\title{
Bilateral Adrenal Hemorrhage due to Sepsis Resulting in Acute Adrenal Crisis
}

Key words: adrenal hemorrhage, acute adrenal crisis

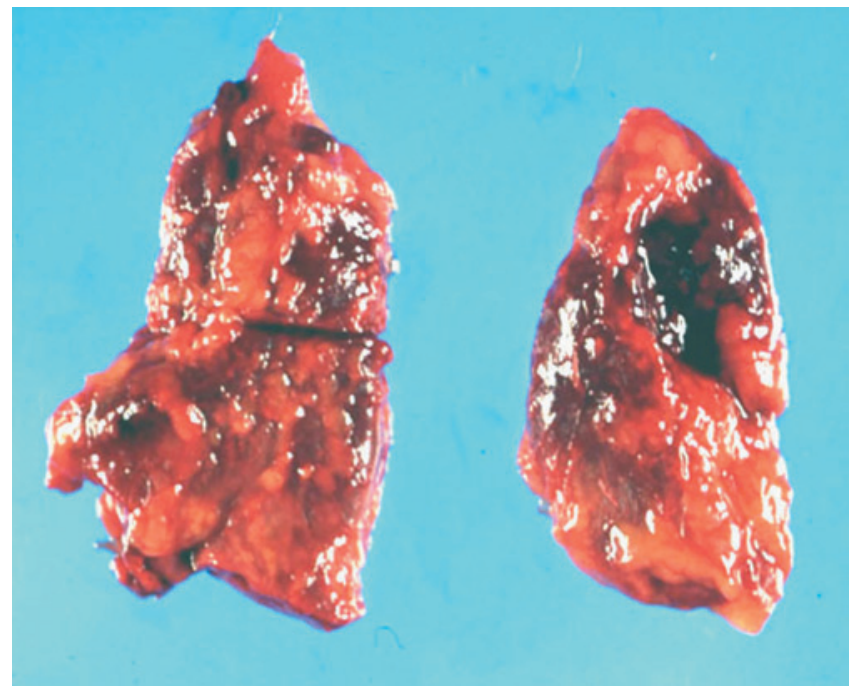

A

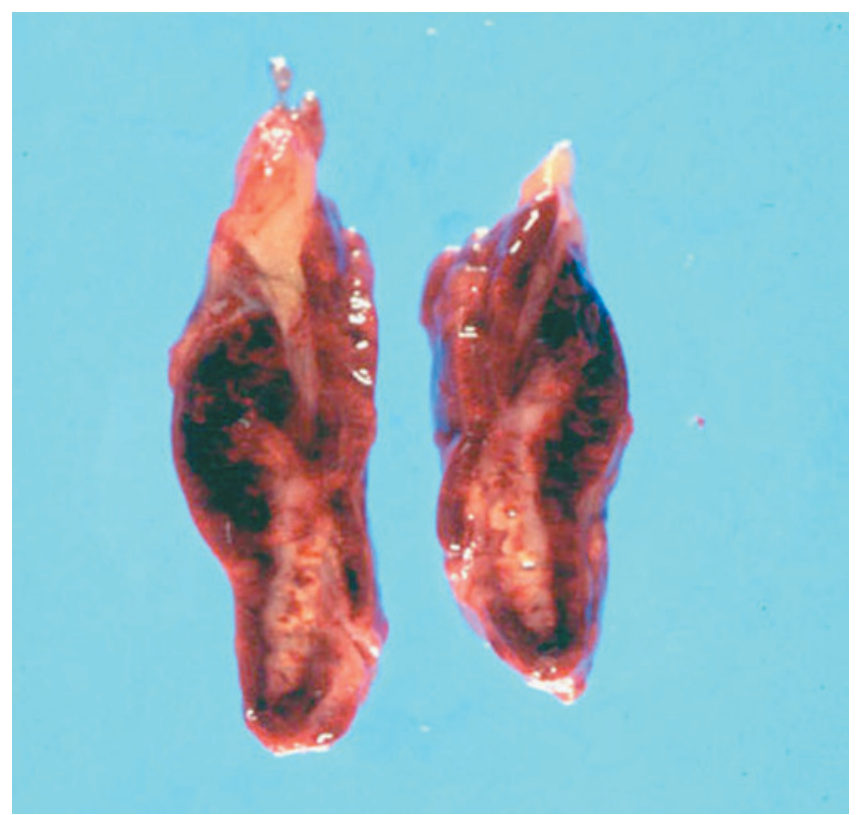

$\mathrm{B}$

Figure 1. The autopsied bilateral adrenal glands were of normal size; however, macroscopic findings showed blackish adrenal cortex (A and B: outer surface and cross-section, respectively).

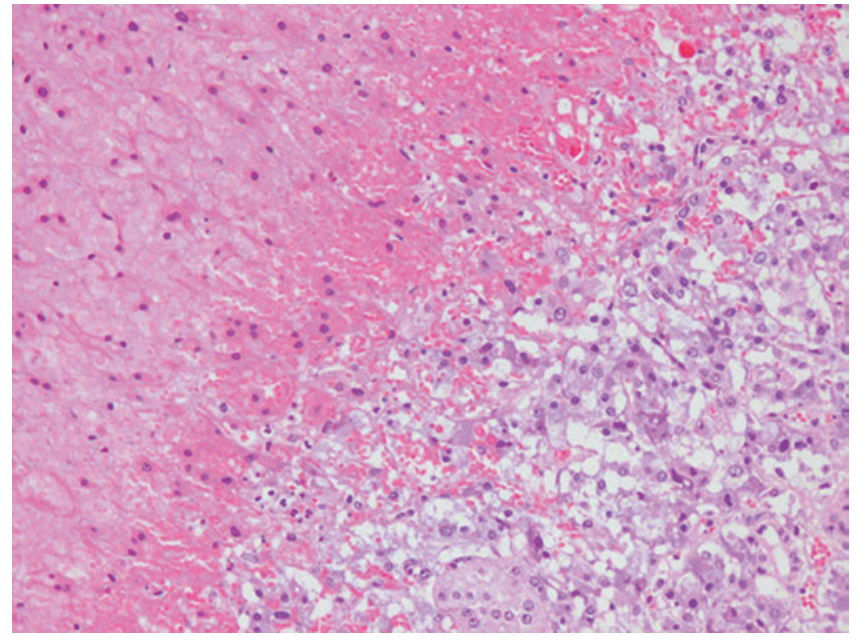

Figure 2. Massive adrenal hemorrhage occurred in the adrenal cortex. Histological examination revealed hemorrhagic necrosis in all zones of adrenal cortex and no abnormal findings in adrenal medulla (HE stain, $\times 200$ ). 
A 77-year-old woman was admitted to our hospital with dysarthria and difficulty in walking on May 28, 2004. As brain CT scan revealed multiple low-density areas, lacunar cerebral infarction was diagnosed. Dysarthria and difficulty in walking were resolved by conservative therapy and rehabilitation. The patient then experienced a sudden rise in body temperature and Klebsiella pneumoniae was found in a blood sample collected from a peripheral artery on June 9. Although the patient was treated with antibiotics and gamma globulin for sepsis, no response was observed. After that she suffered septic shock and loss of consciousness (JCS III-300). Her blood pressure did not respond to the administration of dopamine or norepinephrine, and she died on June 12. During this history, no other symptoms of adrenal failure, such as anorexia, fatigue, nausea, vomiting, progressive hyponatremia or hyperkalemia were observed. However, her serum cortisol level was relatively low: $14.5 \mu \mathrm{g} / \mathrm{dl}$ on the morning on June 12. Macroscopic (Fig. 1) and histological findings (Fig. 2) were consistent with bilateral adrenocortical hemorrhage.

The cause of the patient's death was determined to be acute adrenal crisis due to bilateral adrenocortical hemorrhage by sepsis, one of the most common etiologies of adrenal hemorrhage. In this patient, adrenal CT scan was not performed because representative symptoms of acute adrenal crisis were not observed except for shock. These macro and microscopic findings are of great value to clinical medicine, because pictures of representative adrenal hemorrhage are rare. Adrenal hemorrhage is difficult to recognize clinically and must be considered whenever symptoms such as loss of consciousness, hypotension and shock develop in a patient.

Naoki HiroI, Hisatsugu IshIMORI*, Yukiyo KANEKo, Hideko KIGUCHI**, Tsuneo NAKAMURA* and Mariko HigA

From the Department of Medicine, *Department of Neurosurgery and **Department of Pathology, Saiseikai Kanagawa-Ken Hospital, Yokohama Received for publication February 21, 2005; Accepted for publication May 16, 2005

Reprint requests should be addressed to Dr. Naoki Hiroi, the Department of Medicine, Saiseikai Kanagawa-Ken Hospital, 6-6 Tomiya-cho, Kanagawa-ku, Yokohama, Kanagawa 221-8601 